\title{
Additive aggregation with variable interdependent parameters: the VIP Analysis software
}

\author{
Luis C. Dias ${ }^{*}$, João N. Clímaco \\ University of Coimbra - School of Economics and INESC Coimbra
}

\begin{abstract}
We consider the aggregation of multicriteria performances by means of an additive value function under imprecise information. The problem addressed here is the way an analysis may be conducted when the decision makers are not able to (or do not wish to) fix precise values for the importance parameters. These parameters can be seen as interdependent variables that may take several values subject to constraints. First, we briefly classify some existing approaches to deal with this problem. We will argue that they complement each other, each one having its merits and shortcomings. Then, we present a new decision support software - VIP Analysis - which incorporates approaches belonging to different classes. It proposes a methodology of analysis based on the progressive reduction of the number of alternatives, introducing a concept of tolerance that lets the decision makers use some of the approaches in a more flexible manner.
\end{abstract}

Key words: multicriteria decision analysis, additive value function, imprecise information, decision support systems

\section{INTRODUCTION}

Additive value functions are a well-known approach to rank decision alternatives according to multiple criteria ${ }^{1}$. We consider the use of an additive value function to help Decision Makers (DMs) find the most preferred alternative. Building such a function requires fixing the values of the criteria importance parameters (often called scaling constants or weights). This is one of the most difficult parts of the decision aid process, since these parameters will reflect the DMs' values and trade-offs.

Indeed, not only DMs may find it hard to provide precise figures about their preferences, but also these preferences may change as the decision aid process evolves. Moreover, the procedures that can be used to elicit the values of the importance parameters may require more time and patience than the DMs can spare. DMs may sometimes be unable to separate their intuitive notion of the importance of each criterion from the meaning of the importance parameters, which depends on how the model was structured. Finally, in group decision situations the opinions and preferences of the DMs diverge frequently.

Despite these difficulties, it is usual to expend some resources, such as DMs' time and goodwill, and perhaps even the friendship among themselves, to reach a consensus on the "right" or "best fit" value for each parameter. This leads to a "reference" or "central" result, i.e. a provisional

\footnotetext{
* Corresponding author: Luis C. Dias, Faculdade de Economia, Universidade de Coimbra, Av. Dias da Silva, $3004-512$ Coimbra, Portugal. E-mail: LDias@inescc.pt
} 
best choice. Frequently, DMs feel there is some arbitrariness in the choice of those "right" values and perform an a posteriori sensitivity analysis. This sensitivity analysis usually means to find the parameter variation region where the conclusion of the study remains valid ${ }^{2,3}$ or to identify the "nearest" alternative conclusions ${ }^{4}$.

This kind of analysis has some drawbacks. First, it requires the initial effort of determining an exact value for each parameter. Secondly, the DMs will be led to direct their attention to the "reference" result prematurely. Finally, sensitivity analysis is often conducted varying one parameter at a time, ignoring the interaction effects that could appear when more than one parameter are changed at the same time.

A different approach is to use variable interdependent parameters subject to constraints. The information leading to the constraints is often called "imprecise", "incomplete", "partial" "poor". We will use the expression "imprecise information", meaning that it does not impose a precise combination of values for the parameters. The purpose of the analysis is now the study of all the conclusions that can be drawn from that information. Hence, it becomes a "robustness analysis" as defined by Roy ${ }^{9,10}$. We refer to conclusions instead of results, since the value function provides a ranking that is likely to change (even if partially) when the parameters take different values. Therefore, an analysis focusing on a whole ranking would likely be of little use. Instead, we see the result of the aggregation function as a set of three types of conclusions ${ }^{11}$ :

- an absolute conclusion refers to one alternative $a_{i}$ and a condition that it satisfies independently of all other alternatives (e.g. the value of $a_{i}$ is never lower than 0.7 );

- an unary (relative) conclusion refers to one alternative $a_{i}$ and a condition that depends on other alternatives (e.g. $a_{i}$ is the best alternative);

- a binary (relative) conclusion refers to a relation between two alternatives (e.g. the value of $a_{i}$ exceeds the value of $a_{j}$ ).

This paper has three objectives. The first one is to classify some of the existing approaches that deal with variable parameters (including existing software). This is the subject of the second section, where we argue that every approach has its shortcomings. The third section addresses our second objective, which is to present a possible way of using a sequence of approaches from different classes. In doing this we introduce a concept of tolerance that lets DMs use some approaches in a more flexible manner. Our last objective is to present the VIP Analysis software, meant to deal with

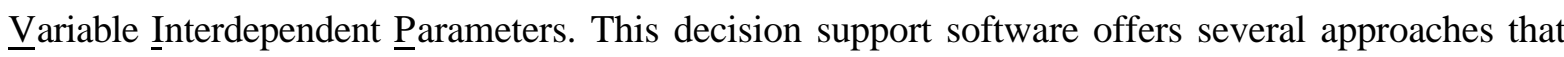
allow an analysis of the decision problem at several levels of detail and from multiple perspectives. The fourth section offers a guided tour of the VIP Analysis and the fifth concludes the paper. 


\section{ADDITIVE AGGREGATION WITH IMPRECISE INFORMATION}

\section{Notation and basic concepts}

In this work we use the following notation:

$A \quad$ Set of alternatives; let there be $m$ alternatives $a_{1}, \ldots, a_{m}$.

$C \quad$ Set of criteria; let there be $n$ criteria.

$k_{j} \quad$ Importance parameter (scaling constant) of the $\mathrm{j}^{\text {th }}$ element of $C$.

$g_{i j} \quad$ Performance of $i^{\text {th }}$ element of $A$ according to the $j^{\text {th }}$ element of $C$.

$T \quad$ Set of acceptable combinations of values for the parameters (each combination is represented as a vector $t \in T$ ) at a given stage of the decision aid process. Let the elements of this set comply with the constraints $k_{j}>0$ and $\sum_{j=1}^{n} k_{j}=1$.

Consider the well-known additive value function: $V\left(a_{i}, t\right)=\sum_{j=l}^{n} k_{j}(t) u_{j}\left(g_{i j}\right)$, where $t$ is an element of the set $T$. Here only the importance parameters $k_{j}(j=1, \ldots, n)$ depend on $t$, although we could consider a more general framework where the shape of the single-objective value functions $u_{j}($.$) and the performances of the alternatives would also be variable interdependent parameters. In$ fact, the extension would be easy if we could vary the performances independently of the scaling constants (which is not too restrictive). We assume that the constraints defining $T$ are linear. They may include bounds $\left(l_{j} \leqq k_{j} \leqq L_{j}\right)$, order constraints (e.g. $k_{1} \geq k_{2}$ ), constraints on trade-offs (e.g. $k_{1} /$ $k_{2} \geq 1.2$ ), constraints implicitly defined through holistic comparisons (e.g. $V\left(a_{1}\right) \leq V\left(a_{2}\right)$ ), etc.

In what follows let $a_{x}$ and $a_{y}$ be any two alternatives in $A$ and let $\operatorname{Opt}\left(a_{x}\right)$ denote the subset of $T$ where the value of $a_{x}$ is higher than (or equal to) the value of the other alternatives in $A$. Let us first recall some concepts of dominance and optimality ${ }^{4,7,12}$ :

- Absolute dominance: $a_{x} \Delta_{\mathrm{A}} a_{y} \Leftrightarrow V\left(a_{x}, t\right) \geq V\left(a_{y}, t^{\prime}\right)$, $\nabla^{\prime} t, t^{\prime} \in T$ and $\exists t, t^{\prime} \in T: V\left(a_{x}, t\right)>V\left(a_{y}, t^{\prime}\right)$.

- Usual (or Bernoulli) dominance: $a_{x} \Delta \mathrm{a}_{\mathrm{y}} \Leftrightarrow V\left(a_{x} t\right) \geq V\left(a_{y}, t\right), \forall t \in T$ and $\exists t \in T: V\left(a_{x}, t\right)>V\left(a_{y}, t\right)$.

- Optimality at $t \in T: a_{x}$ is optimal at $t \Leftrightarrow V\left(a_{x}, t\right) \geq V\left(a_{y}, t\right), \forall a_{y} \in A \backslash\left\{a_{x}\right\} \Leftrightarrow t \in \operatorname{Opt}\left(a_{x}\right)$.

- Potential optimality (p.o.): $a_{x}$ is p.o. $\Leftrightarrow \exists t \in T: \forall a_{y} \in A \backslash\left\{a_{x}\right\}, V\left(a_{x}, t\right) \geq V\left(a_{y}, t\right) \Leftrightarrow \operatorname{Opt}\left(a_{x}\right) \neq \emptyset$.

Dominance refers here to the parameters rather than the multiple criteria and it should be interpreted as a preference relation. Podinovski ${ }^{13}$ suggests the expression "outperforms" instead of "dominates" to prevent confusion. A potentially optimal alternative must be non-dominated (in the usual or Bernoulli's sense).

Next we present briefly some existing approaches, which we divide in four classes. Given a decision problem, one may (and should) use approaches from various classes, since they complement each other. We do not present a thorough chronological review (which may be found, for instance, in references 5, 6 and 12). Most of these approaches consider imprecise information concerning only the 
importance parameters (as we do) whereas few are more general and consider that the performances of the single-objective value functions are also variable.

\section{Approaches based on optimality}

In the context of choosing the most preferred alternative, the solution would be obvious if there was an optimal alternative for all $t \in T$. However, since such an alternative does not usually exist, much of the research in this area has focused on the potentially optimal alternatives.

Rios Insua and French ${ }^{4}$ suggested to elicit an initial set of values for the parameters $t_{0} \in T$ first, leading to a provisional best alternative $a_{0}$. Afterwards, a sensitivity analysis is performed to find the alternatives that are the closer competitors to $a_{0}$. These are the adjacent potentially optimal alternatives to $a_{0}$, i.e. those alternatives $a_{y} \in A$ such that $\operatorname{Opt}\left(a_{y}\right) \cap \operatorname{Opt}\left(a_{0}\right) \neq \varnothing$. They presented a computer tool that encourages the DMs to interactively explore the set of potentially optimal alternatives, by comparing the provisional choice with the potentially optimal alternatives adjacent to it. The remarks that we have made concerning the choice of an initial combination $t_{0}$ remain valid, but lose some strength here due to the interactivity of the approach.

Wolters and Mareschal ${ }^{14}$ proposed to rank the alternatives after choosing a combination $t_{0}$ and a vector distance function. The position of each alternative $a_{y}$ in the ranking is determined by the minimum distance from $t_{0}$ to a vector $t_{y}$ such that $a_{y}$ would be optimal. This approach does not exclude any alternative from consideration, but it requires the choice of an initial combination $t_{0}$.

A family of approaches also based on optimality consists in studying the subsets of $T$ that correspond to different conclusions. Starr ${ }^{15}$ seems to have been the first to suggest this type of approach, in the context of risk decisions with imprecise information on probabilities, followed by other researchers ${ }^{3,16}$.

Starr's "domain criterion" consists in considering the size of the subset of $T$ associated with the optimality of each alternative. Schneller and Sphicas ${ }^{3}$ presented an approximation to this criterion. They take as a starting point a combination $\mathrm{t}_{0}$ and the optimal alternative that corresponds to it. Then, they indicate how to calculate the radius of an (hyper-)sphere centered in to where the alternative remains optimal. Robinson and Soland ${ }^{17}$ suggested to measure the stability of a potentially optimal alternative based on an (hyper-)rectangle of optimality.

Eiselt and Laporte ${ }^{18}$ have suggested the partition of $T$ into several regions, each corresponding to a different potentially optimal alternative, and the computation of their relative volumes. The same idea is present in the work of Antunes and Clímaco $^{2}$ who presented an interactive decision support tool running on Macintosh computers. Its strength lies in its graphical interface, which displays the optimality region of each alternative for problems with two or three criteria. Furthermore, that tool 
allows the visualisation of the regions of $T$ associated with rankings and pairwise comparisons of alternatives.

These approaches, based either on distances or volumes, are usually limited in that they only consider potentially optimal alternatives. Indeed, it is easy to conceive situations with interesting candidates for a best choice that are not potentially optimal. However, Robinson and Soland ${ }^{17}$ propose a flexible notion of optimality, Eiselt and Laporte ${ }^{18}$ suggest other approaches (see 'Pessimistic and aggregation rules' below) and the information that the approach of Antunes and Clímaco $^{2}$ provides to the DMs is not limited to potentially optimal alternatives.

\section{Approaches based on pairwise comparisons}

The class of approaches based on pairwise comparisons builds and exploits a binary relation on the set of alternatives. We start by referring some approaches where these relations are based on the subsets of $T$ that are in favour of each alternative when two alternatives are compared. Weber ${ }^{12}$ defined an "intensity of preference" of $a_{x} \in A$ over $a_{y} \in A$ as the probability of $V\left(a_{x}, t\right) \geq V\left(a_{y}, t\right)$, when taking a random $t \in T$. Bana e Costa ${ }^{19}$ presented an approach for the case with three criteria, where he computes an index for each ordered pair of alternatives $\left(a_{x}, a_{y}\right)$ as the volume of $T$ where $V\left(a_{x}, t\right) \geq V\left(a_{y}, t\right)$. This approach was extended by Bana e Costa and Vincke ${ }^{8}$, where the volume index is compared with other indices. The domains associated with pairwise comparisons for the case with three criteria are graphically displayed by the software from Antunes and Clímaco².

A different subclass of approaches exploits the dominance relation (or a weaker binary relation) in the context of a choice or ranking problem. Kirkwood and Sarin ${ }^{20}$ suggested a ranking of the alternatives based on counting the number of alternatives that dominate it. Park and $\mathrm{Kim}^{6}$ present a different algorithm to rank the alternatives, where dominance is replaced by the "weak dominance" relation proposed by Kmietowicz and Pearman ${ }^{21}$.

There are two recent user-friendly decision support tools running on MS-Windows that belong to the class of approaches based on pairwise comparisons, namely on the notion of dominance. The software PROBE (Preference ROBustness Evaluation), which has been developed under the supervision of Bana e Costa ${ }^{22}$, supports hierarchical criteria and displays a table showing which alternatives are dominated (using symbols and colour). It also computes the range of variation for the value of each alternative (hence it could also be mentioned in the next section), as well as the range of variation of the difference of value between two given alternatives. Its main shortcoming is that it accepts as constraints only a ranking of the importance parameter values. The software DAM (Decision Analysis Module) ${ }^{13}$ is similar to PROBE in identifying dominated alternatives. It also shows the potentially optimal alternatives (using colour). Its main limitation is to consider only interval parameter values, rather than generic constraints. 
The approaches based on pairwise comparisons are able to bypass the "fatal attraction" to optimality. However, the binary relations that these approaches consider are not easy to exploit in a meaningful manner. This is an area where the vast literature on outranking methods ${ }^{9}$ presents many ideas that could be applied in this context.

\section{Approaches based on variation ranges}

A natural approach when using variable parameters is to determine the variation range of some results compatible with $T$, as when we addressed the ELECTRE TRI sorting method ${ }^{23}$. For each alternative $a_{x} \in A$, one may determine the maximum and minimum value that $V\left(a_{x}, t\right)$ may reach, subject to $t \in T$. This allows not only to identify robust conclusions (e.g. the value of $a_{x}$ is never lower than 0.7), but also to know which alternatives are more affected by the fact that parameters are variable. In the context of choosing the most preferred alternative, this type of approach may also enable the elimination of absolutely dominated alternatives.

Butler et al. ${ }^{24}$ suggested the use of Monte-Carlo simulation to find the variation range of the value of each alternative. Their approach also provides other information such as the average value, the standard deviation and several percentiles.

A different approach is to determine the range of variation of the position of an alternative in a ranking. Kampke ${ }^{25}$ discussed the determination of this range in the context of the UTA method, which builds a set $T$ from an holistic ranking performed by the DMs on a subset of alternatives.

Whatever approach is chosen, it will be helpful to consider the use of approaches from other classes since it is likely that many interesting alternatives will exhibit overlapping variation ranges. We believe that the approaches in this class are best suited to an initial screening of a large number of alternatives (in order to reduce that number) before moving on to other techniques.

\section{Pessimistic and aggregation rules}

Some approaches aggregate the values that an alternative achieves for each $t \in T$ into a single figure. Eiselt and Laporte ${ }^{18}$ suggested that this figure could be either the minimum or the average value of each alternative in the domain $T$. These suggestions are also present in the work of Butler et $\mathrm{al}^{24}$, which estimates these figures using Monte-Carlo simulation.

The inspiration for the first approach comes probably from a context where a choice must be made between alternatives whose value varies from scenario to scenario. In that context there are two well-known pessimistic rules: max-min (to choose the alternative that maximises the worst possible outcome) and min-max regret (to choose the alternative that minimises the amount of loss, considering all the scenarios, relatively to each scenario's highest-valued alternative). Kouvelis and 
$\mathrm{Yu}^{26}$ argued the case for the use of these rules in discrete optimisation problems. They sustain that these rules are particularly adequate to non-repetitive decisions and decisions that are evaluated expost by comparing their outcomes with the best possible ones. The same rules may be applied when there is an infinite set of scenarios, which we can consider to be the set $T$.

These approaches are intuitively appealing and provide a ranking of the alternatives. However, they should not be used in isolation, given the information on the worth of each alternative across all the domain $T$ that they disregard.

\section{PROPOSED METHODOLOGY}

\section{Use of multiple approaches}

From the review presented in the second section we can conclude that each approach bears some shortcomings. Indeed, it would be difficult to argue for the superiority of some class of approaches over some other class. Therefore, we believe that several types of approaches should be available during the decision aid process. This would enable the choice of the approaches that better suit the personalities of the DMs, the decision context and the different steps of a decision process.

The range of approaches that may be used at a given circumstance may, however, be constrained by the number of alternatives and the execution time required to produce the results. These two factors are interrelated, since if there are thousands of alternatives, then the computations are likely to take more time. For some approaches, situations with a large number of alternatives may also imply yielding a very large quantity of numbers as output, which may be cumbersome to analyse. We will choose not define how many alternatives are too much for each approach, since this will depend on the tolerance of the DMs to the two factors that we mentioned.

Our purpose is to offer a tool for DMs facing a choice problem, who wish to proceed in their decision process without having to provide precise values for the scaling constants. In this context, we suggest that a decision process should proceed step by step. As the DMs advance onto a new step, the number of alternatives is progressively reduced and the range of approaches that can be used is progressively enlarged. In the meantime, the DMs may be able use the insight that they acquire in the process to provide more information on the scaling constants (i.e. to "narrow" the set $T$ of acceptable combinations of values).

\section{Dealing with a large number of alternatives}

In situations with a large number of alternatives (perhaps thousands), it would be wise to avoid pairwise comparisons, since the number of pairs grows with the square of the number of alternatives. Therefore, an initial analysis should focus on the value range of each alternative compatible with $T$. The ranges may be computed in an exact manner (mathematical programming) or in an approximate 
manner (Monte-Carlo simulation). The latter strategy also yields estimate values for the mean, standard deviation and other statistics.

This type of analysis allows to identify robust conclusions, such as "alternative $a_{i}$ is never worth less than 0.6 " or "alternative $a_{i}$ is never worth more than 0.7 ". If there are absolutely dominated alternatives, then they can be detected and deleted at this stage. Other criteria for reducing the number of alternatives could be to exclude alternatives that may reach a value considered too low by the DMs (as when choosing according to the max-min criterion), or to exclude alternatives that have lower average and higher variance of their value than some other alternative (a criterion often followed in the evaluation of financial investments).

An important information provided by this kind of analysis concerns the width of the interval of value for each alternative, which indicates how much it is affected by the variability of the parameters. There will possibly exist some alternatives that are quite stable, whereas others may exhibit a wide variation range. The DMs' desire of proceeding with a smaller number of alternatives may sometimes be in conflict with their fear of excluding an interesting candidate. For this reason, the software that we will present in the section 'a guided tour of VIP Analysis' allows the provisional exclusion of alternatives, so that they may be re-examined at a later stage.

\subsection{Dealing with a small number of alternatives}

When there is a relatively small number of alternatives the DMs may still use the type of analysis suggested to deal with a larger number. However, the smaller number of alternatives allows the use of approaches based on pairwise comparisons.

We propose in these cases the construction and analysis of an $m \times m$ pairwise comparison matrix that we will represent as $M=\left(m_{i j}\right)$. The elements of this Pairwise Confrontation Table (PCT) will indicate how each alternative compares with each other (in terms of difference of value) under extreme (best and worst) values for the scaling constants. Let us then define:

$$
\begin{aligned}
& \left.m_{i j}=\max \left\{V\left(a_{i}, t\right)-V\left(a_{j}, t\right)\right): t \in T\right\}, \text { for } i, j=1, \ldots, m ; i \neq j, \text { and } \\
& m_{i i}=0, \text { for } i=1, \ldots, m .
\end{aligned}
$$

Hence, $m_{i j}$ is the highest (best case) difference of value of $a_{i}$ over $a_{j}$, whereas $m_{j i}$ is the lowest (worst case) difference.

The computation of all the elements of $M$ requires solving $m(m-1)$ linear programs. However, note that the constraints of these linear programs are always the same: only the objective function changes. The software that we will present below takes advantage of this fact by starting the optimisation at the optimal solution of a prior problem already solved, rather than starting from scratch. This was enough to see our program produce instantaneous results for problems with up to 20 alternatives. Other potentially time-saving strategies, which we have not tried, would be to 
eliminate alternatives as soon as they were found to be dominated and/or to use parallel processing (since the problems could be solved in parallel).

Having calculated $M$, it is possible to draw robust conclusions such as:

- "alternative $a_{i}$ never loses to alternative $a_{j}$ by a difference greater than $m_{j i}$ " (binary conclusion);

- "alternative $a_{i}$ never loses to another alternative by a difference greater than $\max _{\{}\left\{m_{j i}: j=1, \ldots, m\right.$; $j \neq i l$ " (unary conclusion), which is the same as "the maximum loss of opportunity (regret) associated with $a_{i}$ is $\max \left\{m_{j i}: j=1, \ldots, m ; j+i\right\}$ " (see Appendix).

It is also easy to spot dominated alternatives: $a_{i}$ is dominated by $a_{j}$ if $m_{i j} \leq 0$ and $m_{j i}>0$. In the context of a choice problem, the dominated alternatives may be discarded. If the DMs deem that the number of alternatives should be further reduced, then we suggest a more flexible notion of dominance by taking into account a tolerance $s$. Let us define quasi-dominance as follows:

$$
a_{i} \text { quasi-dominates } a_{j} \Leftrightarrow m_{i j}<0 \text { or }\left(m_{i j} \leqq s \text { and } m_{j i}>s\right) \text {. }
$$

Then, the DMs may accept discarding quasi-dominated alternatives when $\varepsilon$ is small, especially if $m_{j i}$ is significantly higher than $\subseteq$.

An optimal alternative, when it exists, is also easily spotted after computing the pairwise confrontation table: $a_{i}$ is optimal if and only if $\max \left\{m_{j i}: j=1, \ldots, m ; j \neq i\right\}$ is negative or null. However, the existence of an optimal alternative will seldom occur before the set $T$ is tight enough. A more useful concept is that of quasi-optimality, which may be defined as follows:

$$
a_{i} \text { is quasi-optimal } \Leftrightarrow \max \left\{m_{j i}: j=1, \ldots, m ; j \neq i\right\} \leq \varrho .
$$

After fixing $\varepsilon$ to an acceptable value, it is straightforward to see if there are any quasi-optimal alternatives (there may exist several). These are the alternatives that never lose to another by a difference greater than the tolerance that was chosen. An alternative approach is to let $\varepsilon$ vary, in order to observe which is the lowest value $\varepsilon$ for which an quasi-optimal alternative exists. If we observe the maximum value in each column of $M$, then this alternative corresponds to the column with a lower maximum. It can be shown that this alternative would be the one chosen by the min-max regret rule (see Appendix).

We believe that the analysis of the Pairwise Confrontation Table $M$, together with the concepts of quasi-dominance and quasi-optimality, will allow DMs to find robust conclusions concerning their search for the best alternative. A more detailed analysis could then be conducted by analysing the volume of $T$ associated with the optimality or quasi-optimality of each alternative (our tool allows this only for the particular case with two or three dimensions), by discussing the constraints defining $T$ that are binding at some of the optimal solutions and by redefining $T$ as new information is provided. We believe that by combining the insight gained by interacting with all of these tools, DMs will be able to 
form a set of convictions that will guide them in providing more information and in choosing the alternative they prefer.

\section{The particular case with two or three dimensions}

In problems with three criteria $(n=3)$ it is possible to represent graphically a $2 \mathrm{D}$ projection of the simplex corresponding to $k_{j} \geq 0$ and $\sum_{j=1}^{n} k_{j}=1$. Antunes and Clímaco ${ }^{2}$ have proposed to display the regions associated with the optimality of each alternative in that triangle. Their approach also allows to visualise the area of the triangle where one alternative is better than some other. This type of approach has the advantage of providing visual feedback to the DMs, since they see those regions, rather than knowing only their relative volume. Another potentiality of this visual feedback is its power as an educational tool. Of course, this type of analysis is also possible when $n=2$, where the projection corresponds to a line segment.

We propose the graphical representation of the region $T$, together with the domains of $T$ associated with the optimality or quasi-optimality of each alternative. It is interesting and pedagogical to observe how the quasi-optimality domain of an alternative enlarges as $s$ increases. Moreover, we are not excluding the alternatives that are not potentially optimal, since every alternative may be quasi-optimal somewhere in $T$ for a sufficiently large $\_$. As a matter of fact, for a given $\Xi$, the relation between the volumes of the optimality domains for some pair of alternatives may sometimes be inverted when considering their quasi-optimality domains.

The flexibility that is brought by the concept of quasi-optimality has a cost, which is the impracticability of displaying the (overlapping) domains of all the alternatives at the same time. Hence, we consider that this approach is most useful when comparing a very small number of alternatives in a pairwise fashion. Each time, we would observe the domains of only two alternatives, possibly visualising also a line dividing $T$ in two regions, one where the first alternative is better than the second and the other where the reverse occurs.

Although there are many decision situations with only three criteria (it is enough to consider the vast literature on bi-criteria problems), this approach is not necessarily excluded in situations with more criteria. First, notice that this approach may be used in situations where it is difficult to set the scaling constants for three criteria, but not for the remaining ones, which may be fixed. Second, note that each equality constraint (e.g. fixing a trade-off rate between two criteria or stating that some two alternatives are worth the same) decreases the dimension of $T$ by one. Indeed, we may use this kind of approach whenever the number of scaling constants that are not fixed minus the number of equality constraints (including $\sum_{j=1}^{n} k_{j}=1$ ) does not exceed two. 


\section{A GUIDED TOUR OF VIP ANALYSIS}

We have built a decision support tool, named VIP Analysis, that implements the methodology

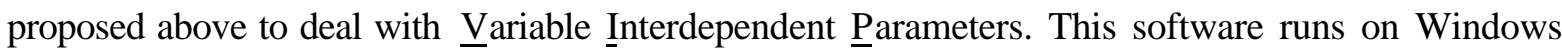
95/98 computers and uses colour extensively. The analyst (or the DMs themselves) will find several approaches in that single package, rather than having to use different programs for different approaches. By implementing several approaches and by giving equal status to all of them, this tool fosters an analysis of the decision situation at multiple levels of detail and from multiple perspectives. Its purpose is to allow the DMs to gain insight on the situation and to let them progress in the decision process without demanding from them precise values for the parameters.

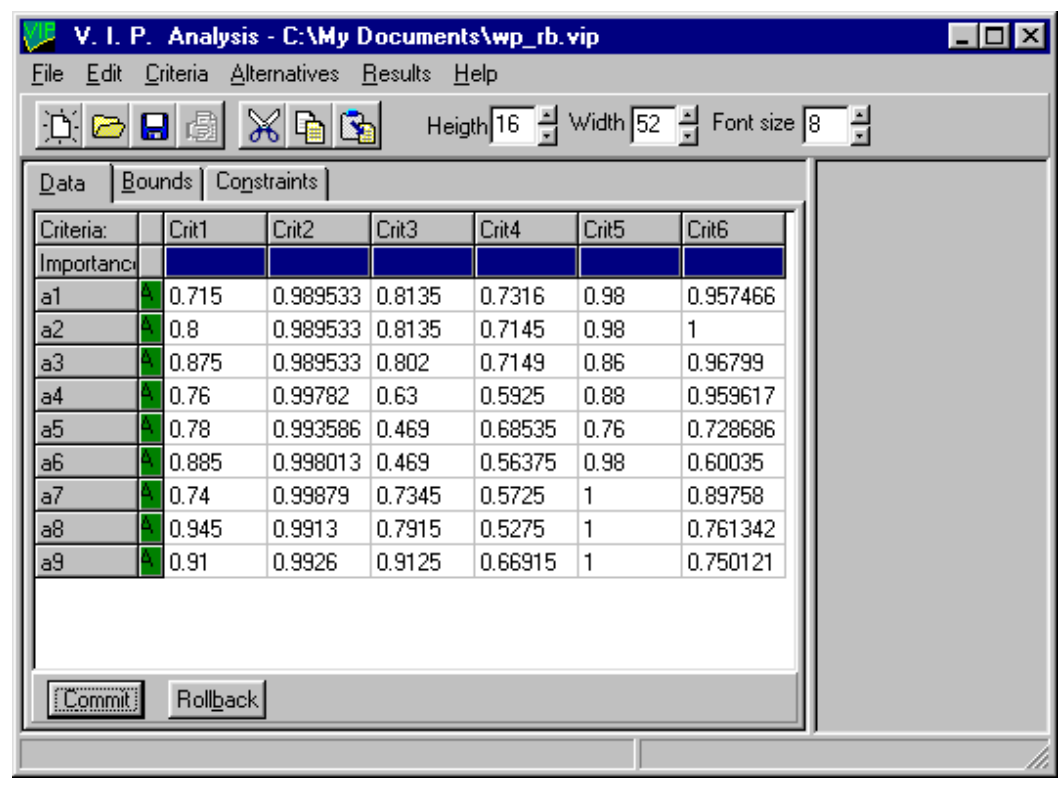

Fig. 1. Table of performances.

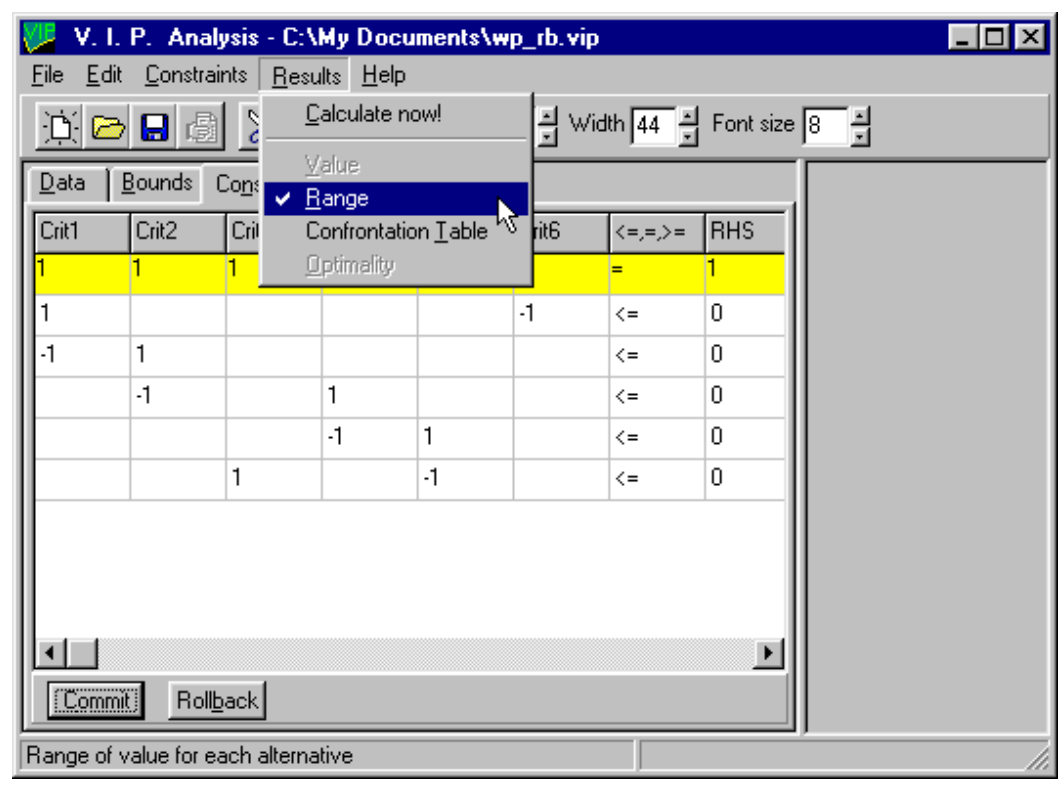

Fig. 2. Constraints defining a ranking of the scaling constants. 
We will start a guided tour of VIP Analysis by considering a situation analysed by Keeney and Nair in the 70s. We follow this study as reported by Roy and Bouyssou', although we will consider additive instead of multiplicative aggregation. This decision situation concerned the choice of a location for a nuclear plant, faced by the Washington Public Power Supply System. Consider the table of performances displayed in Figure 1. There are nine potential sites $\left(a_{1}\right.$ to $\left.a_{9}\right)$ and six criteria: impact on human health (crit1); loss of salmon (crit2); impact on other species (crit3); impact on economy (crit4); aesthetics (crit5); cost (crit6).

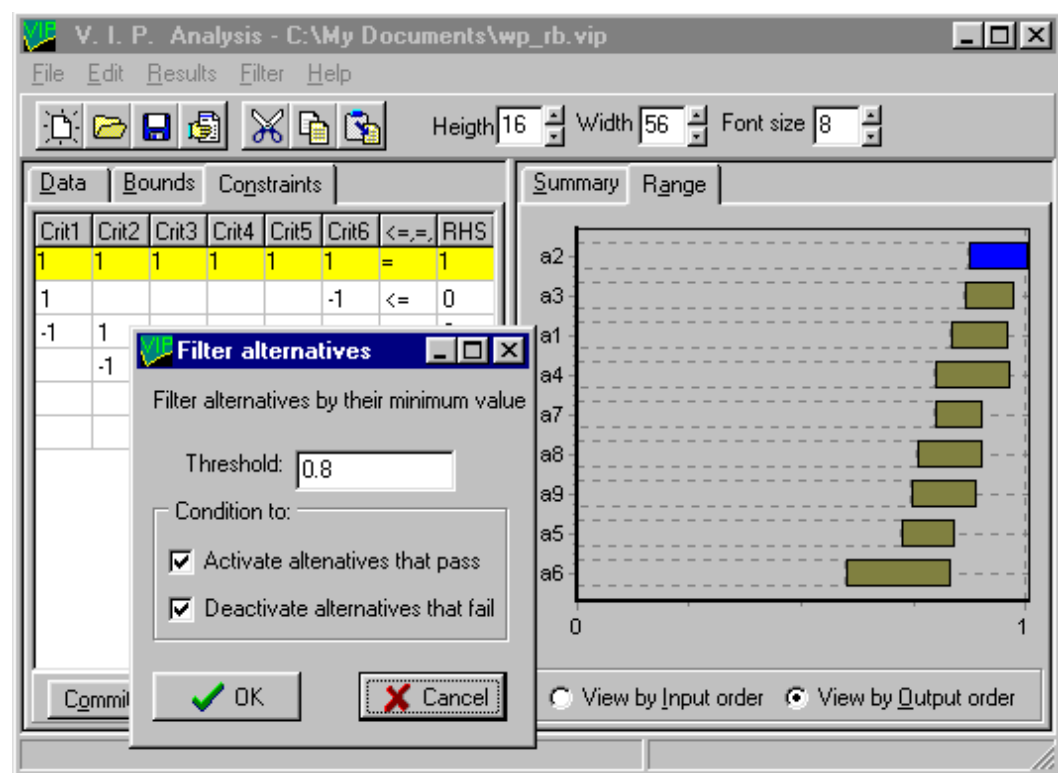

Fig. 3. Filtering by minimum value.

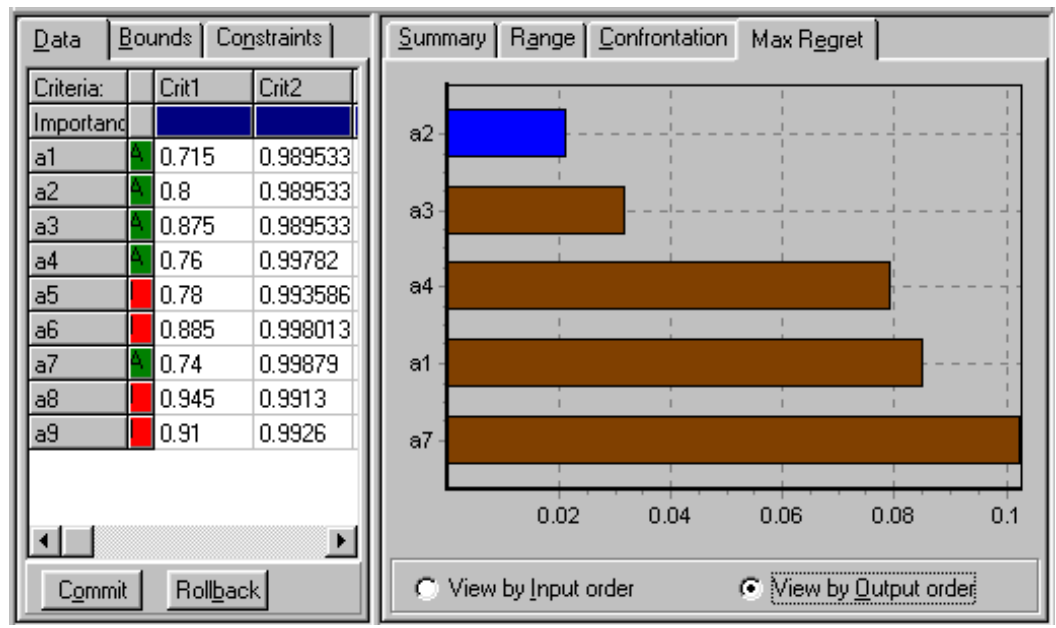

Fig. 4. Bar chart for maximum regret.

The analysts started by asking some questions to the DMs and inferred from their answers the following order for the scaling constants: $k_{6}>k_{1}>k_{2}>k_{4}>k_{5}>k_{3}$. Having VIP Analysis available, let us see how far could anyone go by considering just this piece of information, although not enforcing strict inequalities. As a first approach, let us compute the range of value for each alternative 
(Figure 2). The results show that $a_{2}$ and $a_{3}$ are the best according to the max-min rule (Figure 3 ). The software offers the possibility of filtering the set of alternatives, based on their minimum value, maximum regret or on the possibility of being dominated. In this case, suppose the DMs would pretend to focus on the alternatives with value always higher than 0.8 . The alternatives excluded are marked as inactive but not deleted, so that they may be reactivated later.

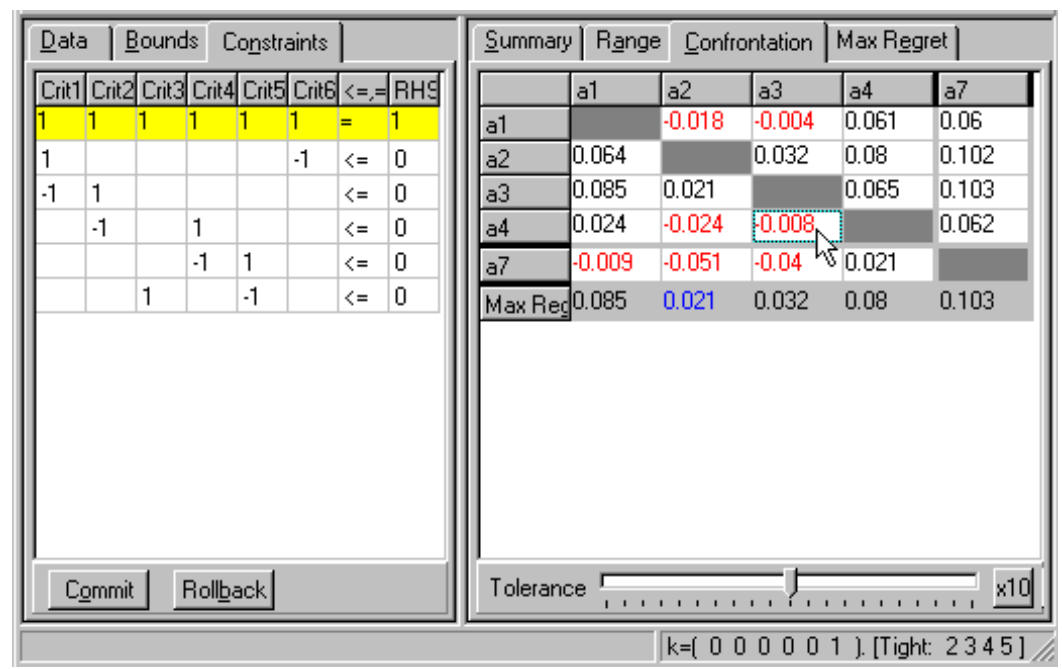

Fig. 5. Pairwise confrontation table.

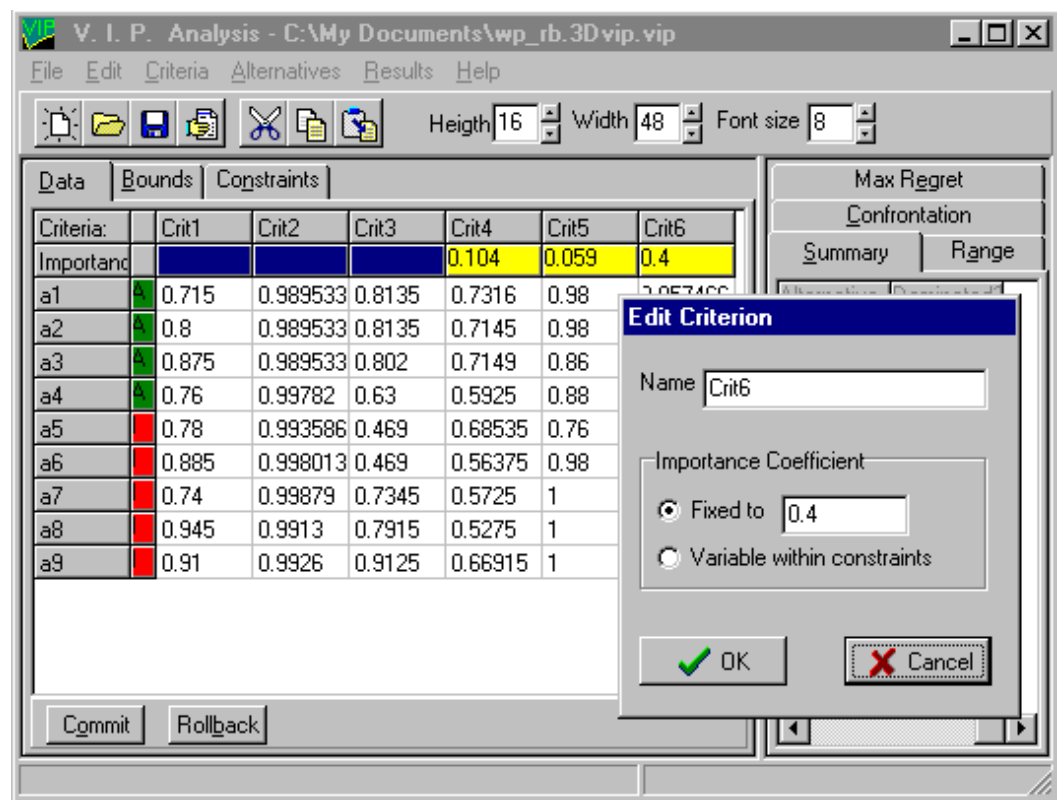

Fig. 6. Fixing the easier trade-offs.

After marking $a_{5}, a_{6}, a_{8}$ and $a_{9}$ as inactive, let us compute the Pairwise Confrontation Table, which also gives us the maximum regret for each alternative (Figures 4 and 5). The negative cells are marked with a different colour indicating that the alternative corresponding to the respective row is dominated by the one corresponding to the respective column. In this situation, only $a_{2}$ and $a_{3}$ are nondominated (Figure 5). When the user selects a cell, the program displays the value of the scaling constants that optimise it, as well as the inequalities that are binding at that optimum (these are the 
constraints that might lead to a different optimum if they were changed). By considering only the constraints $k_{6}>k_{1}>k_{2}>k_{4}>k_{5}>k_{3}$, it is possible to extract some interesting conclusions about $a_{2}$ and $a_{3}$ : they are non-dominated and they are the best two in terms of minimum value and maximum regret. These alternatives happened to be the two with highest value in the original study.

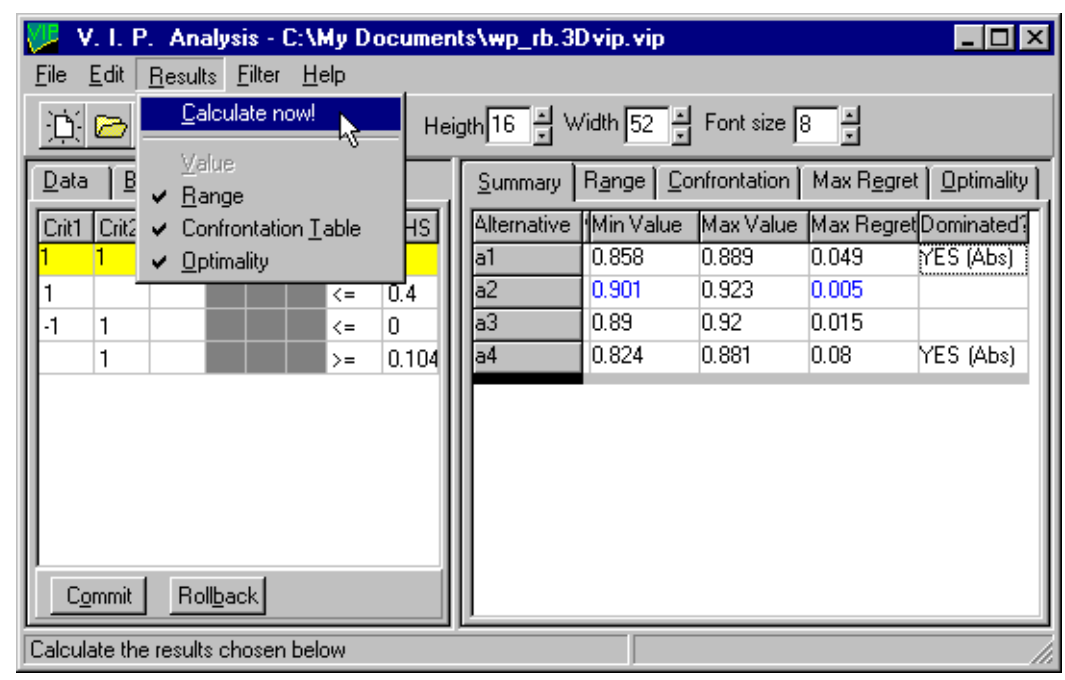

Fig. 7. Summary of the results.

Let us now assume that the DMs were confident enough to answer specific questions about trade-offs between the criteria. In the original study, the cost criterion was considered a standard for the comparisons and its scaling constant was fixed to $k_{6}=0.4$. Then, the DMs traded-off cost for aesthetics and cost for impact on the economy, leading to fix $k_{5}=0.059$ and $k_{4}=0.104$. But now suppose that the DMs would find it much harder to trade-off cost against the remaining criteria (human health, loss of salmon and impact on other species), which is quite plausible. Let us see what would happen if the analysis proceeded without further information, considering only the first four alternatives (Figure 6). Figure 7 displays the modified set of constraints and a summary of the results. Alternative $a_{1}$ is now absolutely dominated, since its best possible value is lower than the worst-case value of $a_{2}$.

To decide between $a_{2}$ and $a_{3}$ let us use an approach that has now become available, given the fact that there are only three criteria that are not fixed: a graphical analysis of their optimality domains. Figure 8 displays the domains of two selected alternatives, plus a line separating the domains where each of the two is better than the other. We can see that the domain associated with the optimality of $a_{2}$ is much wider than the one associated with $a_{3}$. Given this fact, together with the superiority of $a_{2}$ in terms of minimum value and maximum regret, we would pick $a_{2}$ as a quite robust best choice. In the original study $a_{2}$ was ranked second behind $a_{3}$, but by a very small difference. 


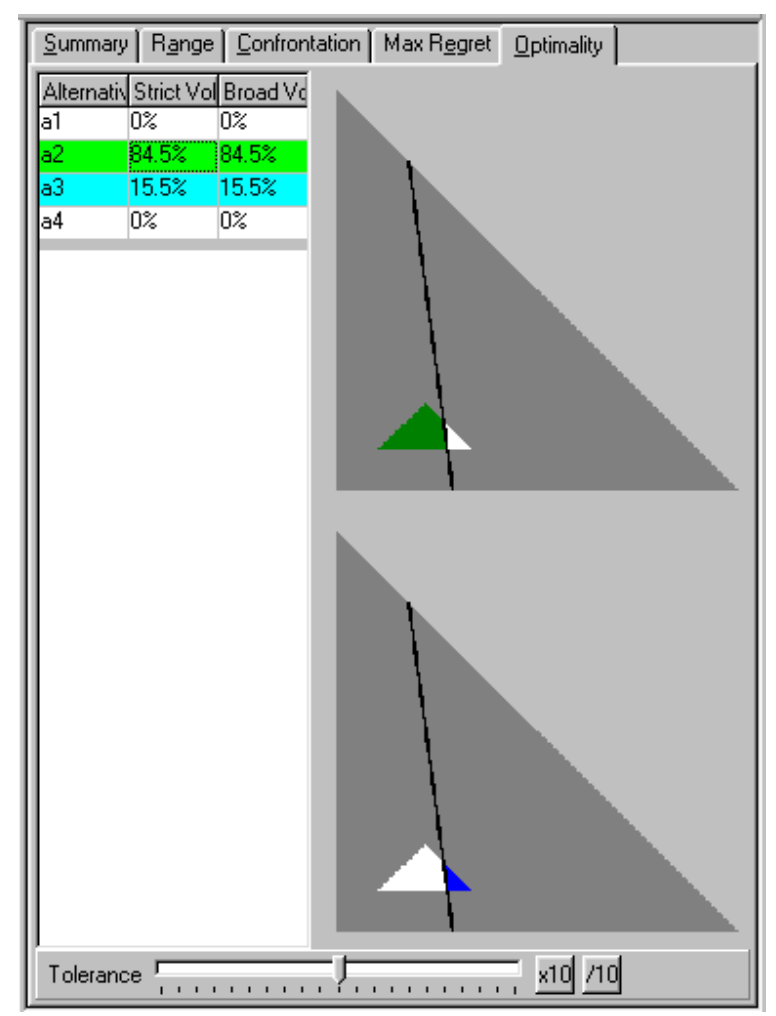

Fig. 8. Optimality domains.

In order to show the importance of allowing a tolerance $\varepsilon$, we will now consider the fictitious situation depicted in Figures 9 and 10. There are now four criteria and the only information available concerns the trade-off between the last two of them $\left(k_{4} / k_{3}=1.2\right)$. This constraint decreases the dimension of $T$, hence allowing its graphical representation. A summary of the results is presented in Figure 11. It can be seen that $a_{4}$ is the alternative least affected by the fact that parameters are variable, whereas $a_{3}$ is the most affected alternative (notice the value ranges). Alternative $a_{4}$ is the best in terms of minimum value and maximum regret.

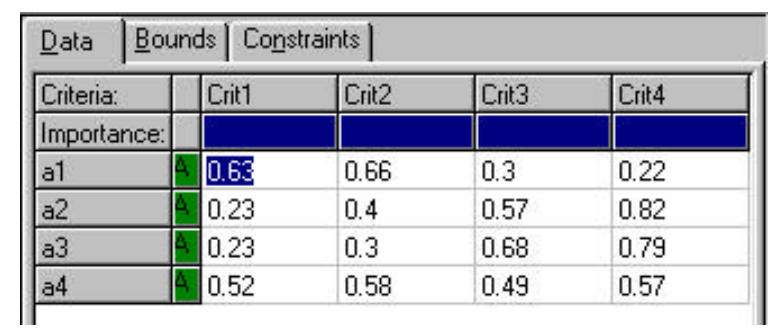

Fig. 9. Data for a fictitious example.

\begin{tabular}{|c|c|c|c|c|c|}
\hline \multirow{2}{*}{$\begin{array}{l}\text { Data } \\
\text { Crit1 } \\
\end{array}$} & \multirow{2}{*}{$\begin{array}{l}\text { Bounds } \\
\text { Crit2 } \\
\end{array}$} & \multicolumn{4}{|c|}{ Constraints } \\
\hline & & Crit3 & Crit4 & $\langle=,=\rangle=$, & $\mathrm{RHS}$ \\
\hline \multirow[t]{2}{*}{1} & 1 & 1 & 1 & $=$ & 1 \\
\hline & & 1.2 & -1 & $=$ & 0 \\
\hline
\end{tabular}

Fig. 10. Constraint defining a fixed trade-off. 


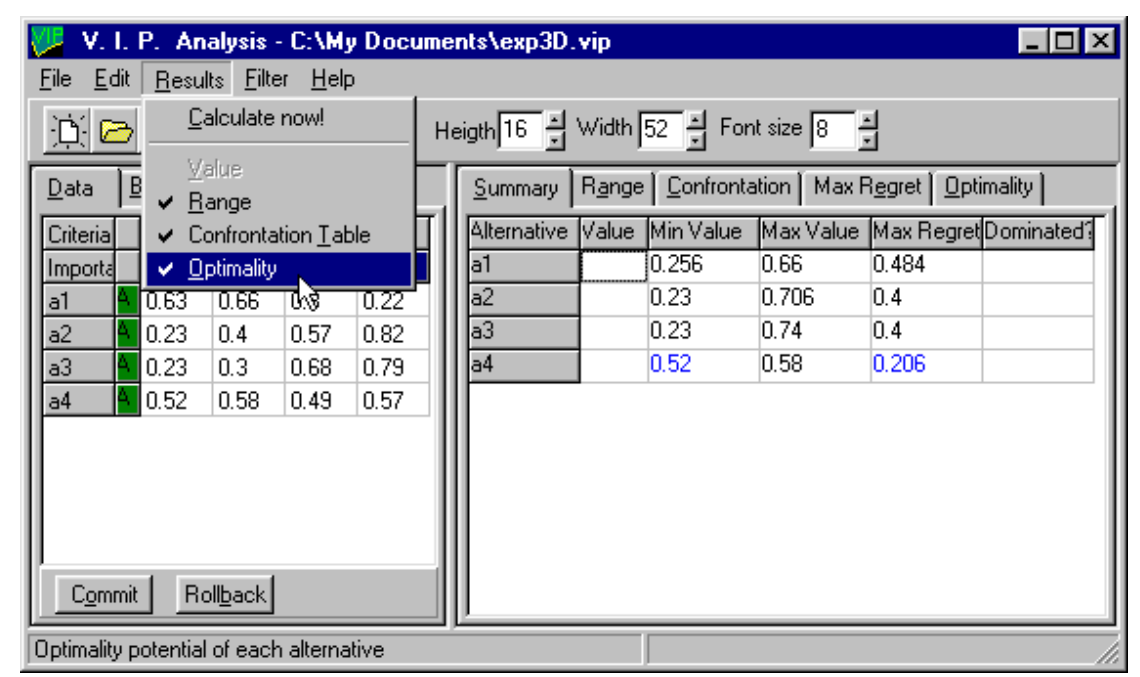

Fig. 11. Summary of the results for the fictitious example.

\begin{tabular}{|c|c|c|c|c|}
\hline Summary $F$ & Range & Confrontation & Max Regret & Qpptimality | \\
\hline & a1 & $a 2$ & a3 & a4 \\
\hline$a 1$ & & 0.4 & 0.4 & 0.11 \\
\hline $\mathrm{a} 2$ & 0.45 & & 0.1 & 0.173 \\
\hline a3 & 0.484 & 0.034 & & 0.206 \\
\hline a4 & 0.277 & 0.29 & 0.29 & \\
\hline Max Regret: & 0.484 & 0.4 & 0.4 & 0.206 \\
\hline $1 \square$ & & & & 1 \\
\hline Tolerance & $\Gamma$ & 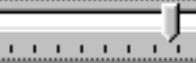 & $=$ & - $\times 1010$ \\
\hline
\end{tabular}

Fig. 12. A situation of quasi-dominance.

The Pairwise Confrontation Table is presented in Figure 12. Let us suppose that the DMs would accept a tolerance of $s=0.07$, which is about $10 \%$ of the maximum value that may be achieved. Under such a tolerance, $a_{3}$ may be considered quasi-dominated by $a_{2}$. Indeed, for all $t \in T$, either $a_{2}$ is "significantly better" than $a_{3}\left(V\left(a_{2}\right)-V\left(a_{3}\right) \geq \varepsilon\right)$ or $a_{2}$ is "almost equal" to $a_{3}\left(\mid V\left(a_{2}\right)\right.$ $\left.V\left(a_{3}\right) \mid \leq \varepsilon\right)$.

Accepting a tolerance also leads to an interesting analysis of the domains where each alternative is optimal / quasi-optimal (Figure 13). Notice how the relation between the relative volumes is inverted when comparing $a_{1}$ with $a_{4}$ (or when comparing $a_{2}$ with $a_{3}$ ). After performing these analyses, it is quite possible that the DMs would be convinced about the merits of $a_{4}$, without needing to provide more information. In fact, $a_{4}$ is the best alternative in terms of minimum value, maximum regret and quasi-optimality domain (when $s=0.07$ ).

When the user clicks on any point in the triangle, the program displays the solution corresponding to it, as well as the value of the two alternatives that are being compared and the value of the optimal alternative at that point. The users may also observe how the domains of quasi- 
optimality change as the tolerance decreases or increases. For all these reasons, we believe that these interactive graphical displays are a powerful tool of analysis and learning.

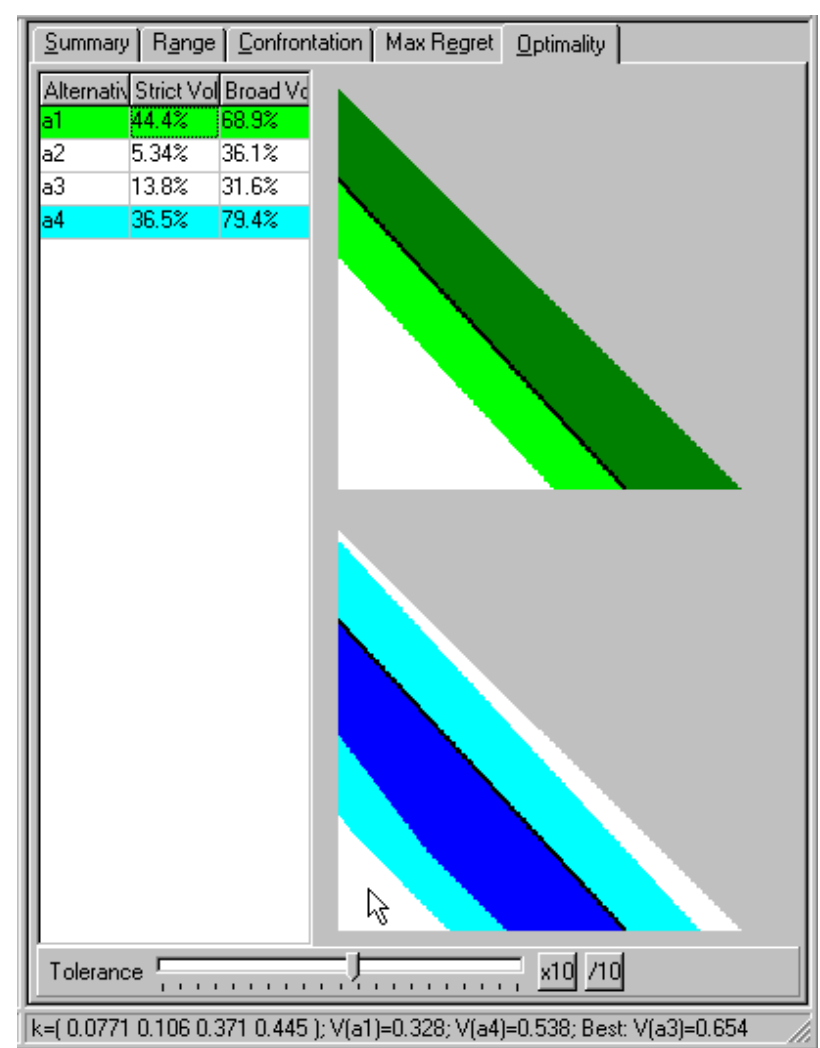

Fig. 13. Optimality and quasi-optimality domains.

\section{SUMMARY AND CONCLUSIONS}

We have presented the main reasons to work with variable interdependent parameters. First, DMs are not forced to find a consensus on the precise values for each parameter. This may save their time, their eagerness to participate and even the friendship between them. Secondly, the conclusions that are drawn (absolute or relative, unary or binary) are robust. Finally, the DMs will know which alternatives are more affected by the variability of the parameters.

After classifying some approaches to deal with imprecise information in additive aggregation, we argued that each one has its merits and shortcomings, and therefore they should be used together. We presented a methodology where multiple approaches are used, providing different perspectives into the decision situation. As the number of candidates for best alternative is progressively reduced, the analysis becomes more detailed as new tools are introduced.

Finally, we presented a new decision support software - VIP Analysis - which fosters the adoption of this methodology by offering a user-friendly interface. VIP Analysis accepts variable values for the scaling constants as well as fixed values. Variable interdependent importance parameters may be constrained by bounds, linear inequalities and linear equalities. The software 
computes the range of value for each alternative, the Pairwise Confrontation Table, the maximum regrets and the graphical display of optimality domains (if the dimension of $T$ allows it). We have shown that both the Pairwise Confrontation Table and the optimality domains are powerful tools to analyse a problem, in particular when the DMs consider a tolerance and the concepts of quasidominance and quasi-optimality.

This type of analysis may provide sufficient arguments for the DMs to agree on a best alternative, or at least on a short list of interesting alternatives, before having to reach exact values for each parameter. Moreover, it may provide them with insight that will help them in the process of agreeing on these values. In the end, no-one will feel the need for a sensitivity analysis. 


\section{APPENDIX: On obtaining the maximum regret from the pairwise confrontation table $M$}

Consider any alternative $a_{i} \in A$. Given some $t \in T$, the regret associated with $a_{i}$ is the difference of value between this alternative and the value of the optimal alternative for $t$. The maximum regret of $a_{i}$ is therefore: $R_{\max }\left(a_{i}\right)=\max \left\{\max \left\{V\left(a_{j}, t\right): j=1, \ldots, m\right\}-V\left(a_{i}, t\right): t \in T\right\}$ $(i=1, \ldots, m)$.

Let us now turn our attention to the pairwise confrontation table $M$, particularly its $i^{\text {th }}$ column. Let $C_{\max }\left(a_{i}\right)=\max \left\{m_{j i}: j=1, \ldots, m ; j \neq i\right\}$.

Proposition: $R_{\max }\left(a_{i}\right)=C_{\max }\left(a_{i}\right)$.

Proof:

a)

Consider the maximum regret of $a_{i}$. There must exist a combination of values $t_{x} \in T$ such that

$R_{\max }\left(a_{i}\right)=\max \left\{\max \left\{V\left(a_{j}, t\right): j=1, \ldots, m\right\}-V\left(a_{i}, t\right): t \in T\right\}=\max \left\{V\left(a_{j}, t_{x}\right): j=1, \ldots, m\right\}-V\left(a_{i}, t_{x}\right)$.

Then, there must exist an alternative $a_{x} E A$ such that $R_{\max }\left(a_{i}\right)=V\left(a_{x}, t_{x}\right)-V\left(a_{i}, t_{x}\right)$.

Now, the element of $M$ where $a_{x}$ is confronted against $a_{i}$ is $\left.m_{x i}=\max \left\{V\left(a_{x}, t\right)-V\left(a_{i}, t\right)\right): t \in T\right\}$,

which must be greater than or equal to $V\left(a_{x}, t_{x}\right)-V\left(a_{i}, t_{x}\right)$.

Since $C_{\max }\left(a_{i}\right)=\max \left\{m_{j i}: j=1, \ldots, m ; j \neq i\right\} \geq m_{x i}$, we conclude that

$$
C_{\max }\left(a_{i}\right) \geq m_{x i} \geq R_{\max }\left(a_{i}\right) \text {. }
$$

b)

Consider the $i^{\text {th }}$ column of $M$. There must exist an alternative $a_{y} \in A$ such that

$$
C_{\max }\left(a_{i}\right)=\max \left\{m_{j i}: j=1, \ldots, m ; j \neq i\right\}=m_{y i} .
$$

Then, there must exist a combination of values $t_{y} \in T$ such that

$$
\left.m_{y i}=\max \left\{V\left(a_{y}, t\right)-V\left(a_{i}, t\right)\right): t \in T\right\}=V\left(a_{y}, t_{y}\right)-V\left(a_{i}, t_{y}\right) .
$$

Hence, $C_{\max }\left(a_{i}\right)=V\left(a_{y}, t_{y}\right)-V\left(a_{i}, t_{y}\right) \leq \max \left\{V\left(a_{j}, t_{y}\right)-V\left(a_{i}, t_{y}\right): j=1, \ldots, m\right\} \subseteq$

$$
\subseteq \max \left\{\max \left\{V\left(a_{j}, t\right)-V\left(a_{i}, t\right): j=1, \ldots, m\right\}: t \in T\right\} \text {. }
$$

Since $\max \left\{\max \left\{V\left(a_{j}, t\right)-V\left(a_{i}, t\right): j=1, \ldots, m\right\}: t \in T\right\}=$

$$
=\max \left\{\max \left\{V\left(a_{j}, t\right): j=1, \ldots, m\right\}-V\left(a_{i}, t\right): t \in T\right\},
$$

we now conclude that

$$
C_{\max }\left(a_{i}\right) \leq R_{\max }\left(a_{i}\right) \text {. }
$$

Finally, joining the conclusions from a) and b) yields: $C_{\max }\left(a_{i}\right)=R_{\max }\left(a_{i}\right)$. 


\section{REFERENCES}

1 Keeney RL and H Raiffa (1976). Decisions with multiple objectives: preferences and value tradeoffs. Wiley: New York.

2 Antunes CH and JN Clímaco (1993). Decision aid for discrete alternative multiple criteria problems: a visual interactive approach. Information and Decision Technologies 19: 185-193.

3 Schneller GO and G Sphicas (1985). On sensitivity analysis in decision theory. Decis Sci 16: 339-409.

4 Rios Insua D and S French (1991). A framework for sensitivity analysis in discrete multi-objective decision-making. Eur J Opl Res 54: 176-190.

5 Athanassopoulos AD and VV Podinovski (1997). Dominance and potential optimality in MCDA with imprecise information. J Opl Res Soc 48: 142-150.

6 Park KS and SH Kim (1997). Tools for interactive multiattribute decisionmaking with incompletely identified information. Eur J Opl Res 98: 111-123.

7 French S and D Rios Insua (1989). Partial information and sensitivity analysis in multi-objective decision making. In Lockett AG and G Islei (eds). Improving decision making in organisations. Springer-Verlag: Berlin, pp. 424-433.

8 Bana e Costa CA and Ph Vincke (1995). Measuring credibility of compensatory preference statements when trade-offs are interval determined. Theory and Decision 39: 127-155.

9 Roy B and D Bouyssou (1993). Aide multicritère à la décision: méthodes et cas. Economica: Paris.

10 Roy, B (1998). A missing link in OR-DA: robustness analysis. Foundations of Computing and Decision Sciences 23, 141-160.

11 Dias LC and JN Clímaco (1999). On computing ELECTRE's credibility indices under partial information. J Multi-Crit Decis Anal (to appear).

12 Weber M (1987). Decision making with incomplete information. Eur J Opl Res 28: 44-57.

13 Podinovski VV (1999). A DSS for multiple criteria decision analysis with imprecisely identified trade-offs. Eur J Opl Res 113: 261-270.

14 Wolters W and B Mareschal (1995). Novel types of sensitivity analysis for additive MCDM methods Eur J Opl Res 81: 281-290.

15 Starr MK (1962). Product design and decision theory. Prentice-Hall: Englewood Cliffs.

16 Charnetski JR and RM Soland (1978). Multiple-attribute decision making with partial information: the comparative hypervolume criterion. Naval Research Logistics 25: 278-288.

17 Robinson MS and RM Soland (1997). The sensitivity analysis of "inexact" multicriteria decisions. In Fandel G and T Gal (eds). Multiple criteria decision making - Proceedings of the twelfth international conference. Springer: Berlin.

18 Eiselt HA and G Laporte (1992). The use of domains in multicriteria decision making Eur J Opl Res 61: 292-298.

19 Bana e Costa CA (1988). A methodology for sensitivity analysis in three-criteria problems: a case in municipal management. Eur J Opl Res 33: 159-173.

20 Kirkwood CW and RK Sarin (1985). Ranking with partial information: a method and an application. Opns Res 33: 3848.

21 Kmietowicz ZW and AD Pearman (1984). Decision theory, linear partial information and statistical dominance. Omega 12: 391-399.

22 CISED Consultores, Lda. (1998). PROBE for Windows - User Manual. Lisbon.

23 Dias LC and JN Clímaco (1998). ELECTRE TRI and group decision aiding: an analytic study on the robustness of the conclusions. Proceedings of the 48th Meeting of the European Working Group “Multicriterion Decision Aid". Quebec. Sept. 24-26, 1998.

24 Butler J, J Jia and J Dyer (1997). Simulation techniques for the sensitivity analysis of multicriteria decision models. Eur J Opl Res 103: 531-546.

25 Kampke T (1996). Sensitivity analysis for assessing preferentially independent order relations. Computers Ops Res 23 : 1119-1130.

26 Kouvelis P and G Yu (1997). Robust discrete optimization and its applications. Kluwer: Dordrecht.

This research has been partially supported by Technological Development and Scientific Research contract PRAXIS/PCSH/C/CEG/28/96. 